\title{
Aproximaciones a la problematización del objeto digital en el marco de la digitalización de la vida
}

\author{
Marcelo J. García Farjat y Sergio W. Salguero ${ }^{1}$
}

Recibido: 01/04/2019; Aceptado: 22/04/2019

Cómo citar: García Farjat, M. y Salguero, S. W. (2019). Aproximaciones a la problematización del objeto digital en el marco de la digitalización de la vida. Revista Hipertextos 12(7), pp. 47-67. DOI: https://doi.org/10.24215/23143924e003

\section{Resumen}

El presente trabajo tiene como objetivo aproximarse a los objetos digitales, la pregunta por su materialidad y su modo de existencia como realidades presentes y desarrolladas en nuestra cotidianeidad digitalizada. La posibilidad de explorar las problematizaciones del objeto digital, en el marco del fenómeno creciente de digitalización, marca un impacto significativo en la existencia a partir de un supuesto empobrecimiento de la experiencia humana y de la constitución de una nueva subjetividad. Algunos interrogantes que permiten pensar estos objetos digitales y que van a guiar el artículo son los siguientes: ¿cómo pensar la existencia de los objetos digitales?, ¿cómo aproximarnos a pensar la materialidad de los objetos digitales más allá de su instrumentalidad? Desde estos interrogantes se analizan algunas posiciones esencialistas y se recuperan aspectos que apuntan a una materialidad expresada en las relaciones entre estos objetos más que a su concreción.

Palabras claves: objetos digitales, experiencia humana, digitalización.

\begin{abstract}
The objective of this paper is to approach digital objects, their substance and limits, taking into account that they are present and well-developed realities in our digitalized everyday life. The possibility of exploring the problematic of the digital objects in the context of the growing phenomenon of digitalization has a significant impact on society from a supposed impoverishment of human experience and the constitution of a new subjectivity. Some questions that allow us to
\end{abstract}

\footnotetext{
${ }^{1}$ García Farjat, Marcelo José. Lic. en Comunicación Social. Maestrando en Tecnología, Políticas y Culturas, Centro de Estudios Avanzados (CEA). Universidad Nacional de Córdoba. Córdoba, Argentina. garciaf_marcelo@live.com.

Salguero, Sergio Walter. Prof. en Filosofía y Ciencias de la Educación. Especialista en Educación y TIC. Maestrando en Tecnología, Políticas y Culturas, Centro de Estudios Avanzados (CEA). Universidad Nacional de Córdoba. Córdoba, Argentina. filosergiosofia@gmail.com.
} 
think about these digital objects are the following: How do we think about the existence of digital objects? How do we approach reasoning the materiality of digital objects beyond their instrumentality? Considering these questions, some essentialist positions are analyzed, and aspects that point to a materiality expressed in the relations between these objects are recovered rather than their concretion.

Key words: digital objects, human experience, digitalization.

\begin{abstract}
Resumo
O presente trabalho tem como objetivo abordar objetos digitais, questionar sua materialidade e seu modo de existência como realidades presentes e desenvolvidas em nosso cotidiano digitalizado. A possibilidade de explorar as problematizações do objeto digital, no marco do crescente fenômeno da digitalização, marca um impacto significativo na existência, decorrente de um alegado empobrecimento da experiência humana e da constituição de uma nova subjetividade. Algumas perguntas que nos permitem pensar sobre esses objetos digitais e que orientarão o artigo são: como pensar sobre a existência de objetos digitais? Como podemos abordar a materialidade dos objetos digitais além de sua instrumentalidade? A partir dessas questões, analisam-se algumas posições essencialistas e recuperam-se aspectos que apontam para uma materialidade expressa nas relações entre esses objetos e não na sua concretização.
\end{abstract}

Palavras chave: objetos digitais, experiência humana, digitalização.

\title{
1. Introducción
}

\section{[...] corremos el riesgo de no preguntarnos si nuestro modo de ser hombres} no es demasiado antiguo para habitar la era de la técnica.

(Galimberti, 2001:37)

La informatización de la sociedad es un proceso que viene desarrollándose a una velocidad exponencial configurando cambios en los vínculos que establecemos con la técnica. La llamada sociedad de la información aparece como un marco o escenario con pretensiones de justificar innovaciones educativas, prácticas políticas y sociales hasta la necesidad de pensar una antrobología o una nueva condición humana. Así lo expresa Eric Sadin (2017), citando a Jacques Ellul, recordando que:

La Técnica es, de hecho, el medio ambiente del hombre. Estas mediaciones se han generalizado, extendido, multiplicado de tal modo que 
han terminado por constituir un nuevo universo... Hemos visto aparecer el 'medio técnico' (...) Ya no vive en contacto con las realidades de la tierra y del agua, sino con aquellos instrumentos y objetos que conforman la totalidad de su entorno (Sadin, 2017: 43).

Esta realidad marcada por la presencia de la técnica desequilibra de algún modo el perfil instituido para el pensador de la época: "la idea del filósofo como una figura que sobresale como mero crítico y defiende la pureza del pensamiento se ha borrado en el flujo del progreso tecnológico" (Hui \& Lovink, 2016:1). Sin embargo, mientras diversos abordajes teóricos ingenieriles o instrumentalistas sostienen haber cubierto todas las aristas de la revolución tecnológica, aquello que impulsa precisamente esta revolución, el software, no ha sido evidenciado en sus efectos culturales y sociales: "Si no atendemos al software mismo corremos el peligro de siempre tratar con sus efectos más que con las causas: la salida que aparece en una pantalla de computadora más que los programas y culturas sociales que producen esas salidas" (Manovich, 2008: 4).

El presente trabajo busca aproximarse a la problematización de los objetos digitales entendidos como realidades presentes y desarrolladas en un mundo cotidiano digitalizado comprendido por mensajes convertidos en una sucesión de impulsos eléctricos, transformados en código binario generando diversos objetos digitales. La informatización le agrega a este proceso de digitalización órdenes y operaciones recursivas que transforman y mezclan expresiones previamente digitalizadas. Estos objetos, de carácter ubicuo, hacen difícil proponer una clara (y ya clásica) separación entre lo online y lo offline (Hui, 2017). El giro computacional (Berry, 2011a) y la mediatización intensiva de este entorno nos encuentra con una "tecnologización expansiva de las existencias", provista de múltiples objetos digitales, en numerosas esferas de la vida. Textos, imágenes, sonidos, redes, son más que datos o metadatos o entidades abstractas. En este marco, surgen interrogantes que orientarán las reflexiones sobre los objetos digitales: ¿Cómo pensar la existencia de estos objetos digitales? ¿Cómo abordar su materialidad más allá de su instrumentalidad? Y desde estas preguntas indagar si todo proceso de digitalización causa un empobrecimiento de la experiencia humana. Será preciso revisar cierta concepción de la materialidad focalizada en la pregunta por la sustancia. Y a partir de la pregunta por lo digital o el modo de existencia de lo digital, reconocer aquellos marcos referenciales que abran la reflexión hacia la relevancia de las relaciones entre dichos objetos más que su concreción. 
El recorrido propone, en primer lugar, indagar ciertos aspectos de los objetos digitales, principalmente desde la perspectiva fenomenológica de Hui, que permite pensar la sustancia y los límites del objeto digital ya que "los objetos son siempre objetos de experiencia" (Hui, 2017:81). Más aún si la filosofía, en el olvido de los objetos técnicos (y también diríamos, digitales), fracasa en "absorber el rápido desarrollo de la tecnología y el cambio social después de la revolución industrial” (85). Para ello, es necesario presentar algunos problemas que traen consigo posturas sustancialistas e instrumentales para entender la materialidad de los objetos digitales en el marco actual de la digitalización de la vida.

En este sentido se recuperan algunas advertencias que el propio Hui realiza al expresar que los objetos digitales no son simplemente unidades básicas de información expresadas en código binario, como lo propone la física digital o la ontología digital de E. Fredkin y S. Wolfram. Luego, retomando aportes de G. Simondon, se analizan las dificultades de ciertas posiciones esencialistas que afectan en la construcción de la relación cultura-técnica. En el desarrollo de la discusión, se presenta una aproximación al fenómeno de la digitalización y su impacto en la existencia, desde los aportes de E. Sadin y D. Berry, intentando escapar de los determinismos tecnológicos y reconociendo los movimientos de constitución de una nueva subjetividad. Para concluir, se intentará responder a los interrogantes planteados en esta introducción.

\section{Una aproximación a la materialidad de los objetos digitales}

La presente búsqueda se orienta a proponer un abordaje filosófico que tenga por objetivo identificar la realidad a la que se refieren los objetos digitales. La sola crítica cultural de la tecnología es insuficiente para la comprensión del fenómeno tecnológico. La experiencia con estos objetos configura la impresión generalizada de una apertura infinita y constitutiva de lo digital "que supone juegos combinatorios ilimitados vinculados con el aumento exponencial de los poderes de almacenamiento y tratamiento de los datos y con la variedad creciente de los tipos de datos disponibles" (Sadin, 2018: 27).

En este sentido, y a partir de la comprensión de sus implicancias en la vida "real”, será imprescindible avanzar hacia marcos teóricos que vayan más allá del 
inicial concepto de lo "virtual", concepto en boga hace unos años que se vinculaba a algunas comunidades y tipo de interacciones en medios digitales (Hui, 2016: 47). Ahora, "si la ontología comienza con la cuestión del ser, hay un problema si la comprensión del ser no está en el camino correcto, si esta no toma en cuenta la naturaleza de la tecnología” (Hui, 2017: 86). En la línea de la filosofía digital, y si bien los aportes de Fredkin (2003) son relevantes en términos físicos y computacionales, su abordaje atomista presenta limitaciones para tratar con el aspecto relacional de los objetos digitales al considerar que todo se basa en algún simple proceso discreto, bajo coordenadas espacio-temporales, y que todo cambio de índole informacional es una consecuencia de procesos informacionales digitales.

Asimismo, es posible descartar teorías duales que definen a los artefactos como estructuras físicamente diseñadas con determinadas funciones regidas por las intenciones de los seres humanos. Bajo esta órbita, el enfoque instrumentalista, en el desarrollo de una ontología de los artefactos, presenta ciertas limitaciones que dificultan la cabal comprensión de los objetos técnicos y digitales. La llamada "teoría de la instrumentalización” de Feenberg (2005), toma algunos aportes, por un lado, de filósofos como Heidegger y Marcuse, y por otro, de los estudios empíricos en ciencia y tecnología, que dan cuenta de las dificultades que trae consigo un enfoque instrumentalista para explicar y aprehender los desarrollos tecnológicos y su vinculación a las distintas sociedades. Esto responde, por un lado, a la proclamación de la neutralidad de la tecnología (la tecnología como un elemento puro), y por otro, a la asociación de este concepto a las herramientas; a lo meramente artefactual. Así, la tecnología se halla en un plano externo que permea los cambios sociales, se presenta avalorativa, acrítica y universal, siguiendo un camino racional, ordenado y autónomo. Esta concepción, extendida a los objetos digitales, permite advertir que si se constriñen a sus funciones predeterminadas, a lo que hacen los humanos con "intención” y "significado" (Latour, 2008), se soslayan aspectos esenciales que comprenden a estos como datos y procesos que producen agencia a partir de codificaciones:

...estamos tratando con "acciones de software"- ya que es un software el que define las opciones para navegar, editar y compartir el documento, en lugar del propio documento... Mientras que el contenido del archivo, obviamente, forma una parte de esta experiencia, está también formada por la interfaz y las herramientas proporcionadas por el software (Manovich, 2008: 14). 
Es necesario, siguiendo el planteamiento de la teoría del Actor-Red, proponer un tratamiento de carácter simétrico entre objetos y seres humanos 2 , a fin de dar cuenta sobre quién y qué participa en la acción (Latour, 2008). Será preciso descartar nociones que colocan en un segundo plano el papel de los objetos digitales para la comprensión de las tecnologías digitales en la cotidianeidad y con ello, la consecuente construcción de nuevas subjetividades. También será deseable rechazar propuestas de abordaje que limiten la concepción de los objetos digitales a los significados circunscriptos al uso que disponen (y dispondrán) para la acción humana, y que fuera de ese marco semántico, estos objetos serían inaprensibles. Las cosas, expresa Latour (2008), podrían, más allá de "determinar" y servir como "telón de fondo para la acción humana", ser capaces de autorizar, permitir, hacer posible, prohibir, etc. Cabe aclarar que esto no implica (desde esta perspectiva teórica) que los objetos reemplacen o desenvuelvan las actividades que, en apariencia, competen únicamente al ser humano, no se trata de otorgar privilegio epistemológico a los objetos. Por el contrario, se destaca la necesidad de no minimizar la agencia de los objetos en el contexto del estudio de la durabilidad y extensión de las distintas interacciones, en este caso, digitales.

Así, y en el marco de la ubicuidad de las tecnologías digitales, resulta necesario redefinir o repensar el modo de existencia de los objetos digitales y su interacción con los sujetos. Mientras muchos perciben lo digital en términos de abstracciones (información, datos, metadatos), surge la posibilidad de pensar la materialidad de estos objetos desde el concepto de relaciones (Hui, 2017) concretizadas en lenguajes de programación y en ontologías web.

Lo que llamo objetos digitales son simplemente objetos en la web, tales como videos en YouTube, perfiles de Facebook, imágenes en Flickr, y demás, que están compuestos de datos y formalizados por esquemas u ontologías que pueden generalizarse como metadatos. Estos objetos permean nuestra vida cotidiana en línea... (Hui, 2017: 82).

Sabemos que el mundo consiste en átomos, pero pensarlo solo en estos términos no colabora a explicarlo. De manera análoga, una ontología digital

\footnotetext{
${ }^{2}$ Esto, en términos latourianos, implica no imponer a priori una asimetría espuria entre la acción humana intencional y un mundo material de relaciones causales (Latour, 2008: 113).
} 
reducida al código binario ${ }^{3}$, entendida como bits y bytes ${ }^{4}$, no resulta suficiente para alcanzar una comprensión de nuestra vida diaria en medio de la aceleración tecnológica. Al respecto, cabe preguntarse: ¿Cómo pensar la sustancia de un objeto digital? ¿Cómo pensar, además, los límites de un objeto digital? Estos objetos son percibidos por los usuarios de diversas maneras: archivos de texto, códigos binarios, señales de valores de voltaje y operaciones de entradas lógicas, pero "este tipo de reduccionismo no nos dice mucho sobre el mundo" (Hui, 2017:88). Independientemente de los modos de actualización del código (texto, imagen, sonido, programas), los objetos digitales pueden ser considerados "entidades discretas" recortadas del continuo del universo ${ }^{5}$. Y estas entidades acopladas al medio asociado, también digital, en el cual se concretizan ${ }^{6}$ (Berti y Blanco, 2013).

Para considerar el modo de existencia de los objetos digitales de manera relacional habría que tener ciertas precauciones que vienen dadas por considerar el medio asociado al que se acoplan y que puede cambiar:

Una cadena de bits puede realizarse físicamente de las maneras más diversas, pero su significado como objeto digital depende de la interpretación que hagan de sí los programas de su entorno digital. Dichos programas también son objetos digitales, y por lo tanto admiten también múltiples realizaciones; y también son identificados en su propio entorno o medio asociado (Berti, 2017: 20).

La trascendencia del objeto encuentra sus obstáculos o se debilita, no solo en los objetos técnicos ${ }^{7}$ (aunque aquí todavía puede pensarse la sustancia de lo

\footnotetext{
${ }^{3}$ Manovich (2008: 14) expresa: “...incluso si un programa es relativamente corto y un crítico entiende exactamente lo que se supone que el programa hace examinando el código, esta comprensión de la estructura lógica del programa no puede ser traducida a imaginar la experiencia real del usuario. En resumen, estoy sugiriendo que los "estudios de software" no deben confundirse con "estudios del código".

${ }^{4}$ Ya lo advierte Hui (2017: 82) al expresar que los objetos digitales no son simplemente unidades básicas de información expresadas en código binario, como lo propone la física digital o la ontología digital de Edward Fredkin y Stephen Wolfram.

5 Es lo que Stiegler (2002: 36) denominaría como exteriorización: "La técnica, como "proceso de exteriorización", es la continuación de la vida por otros medios que la vida".

${ }^{6}$ Simondon encuentra entre el objeto técnico y el medio natural un medio tanto externo como interno que otorga los requisitos necesarios para su funcionamiento (incluye otros objetos técnicos, máquinas o seres humanos). A este medio lo llama medio asociado: "Se puede afirmar entonces que la individualizaciòn de los seres técnicos es la condición del progreso técnico. Esta individualización es posible por la recurrencia de la causalidad en un medio que el ser técnico crea sí mismo y que lo condiciona tanto como se ve condicionado por él. Este medio, a la vez técnico y natural, se puede denominar medio asociado" (Simondon, 2007: 77-78).

${ }_{7}$ Definir el objeto técnico es una tarea compleja por cuanto su modo de existencia admite tres niveles: elementos, individuos y conjuntos. Este modo de existencia requiere, entre otras etapas, comprender la génesis de los objetos técnicos donde se puede observar el paso de un modo de ser abstracto a un modo de ser concreto. Si bien Hui, de alguna manera considera el objeto digital como un objeto técnico en tanto admite diversos
} 
material), sino específicamente en los objetos digitales ya que cualquiera "puede hacer y destruir estos objetos al presionar unas teclas en un teclado o hacer clic en un mouse" (Hui, 2017:94) pensándose casi, como constitutivos de una materialidad efímera. Por esto al pensar a los objetos digitales en términos de relación se cuestionan visiones sustancialistas ${ }^{8}$. Así, y valorando el concepto de relación, Simondon expresaba que existe "una vía sustancialista, que considera el ser como consistente en su unidad, dado a sí mismo, fundado sobre sí mismo, inengendrado, resistente a lo que no es él mismo, y una vía hilemórfica, que considera al individuo como engendrado por el encuentro de una forma y de una materia" (2015: 7). El concepto de relación es central en la teoría de la individuación de Simondon, para quien adquiere estatuto ontológico, contrariamente a la línea que, desde Aristóteles, ubica a la sustancia como principio fundamental en la constitución de la realidad individual.

En el marco de la propuesta fenomenológica de Husserl (2008), y específicamente en su obra "Crisis de las ciencias europeas y la fenomenología trascendental", se advierte que las formas de las tareas y métodos que comprenden a las distintas ciencias se han vuelto cuestionables. El auge positivista, basado en la matematización y en un empirismo radical para el abordaje de los fenómenos, generó la pérdida de preguntas decisivas de una auténtica humanidad; preguntas ponderadas como metafísicas, incluidas las "oscuramente" llamadas preguntas supremas y últimas: “...esta ciencia no tiene nada que decirnos. Justamente, ella excluye por principio las preguntas que, en nuestros desdichados tiempos, son candentes para los seres humanos abandonados a perturbaciones fatales: las preguntas por el sentido o el sinsentido de toda esta existencia humana" (p. 50).

Hui (2017) sostiene que el trabajo de Husserl está en contra de un realismo ingenuo o relativismo, base de aquella tesis que sostenía una actitud natural,

grados de concreción, su identidad se diferencia del objeto técnico en que no resulta de una concreción física: "El objeto técnico existe entonces como tipo específico obtenido al término de una serie convergente. Esta serie va del modo abstracto al modo concreto: tiende hacia un estado que haría del ser técnico un sistema enteramente coherente consigo mismo, enteramente unificado" (Simondon, 2007: 45).

${ }^{8}$ El planteamiento de Pierre Lévy (1995) podría considerarse un antecedente interesante en cuanto a la concepción de lo digital en términos de relación. En su momento, y al hablar de la virtualización, ya comenzaba a pensar lo objetivo-subjetivo en tanto acontecimientos vinculados recíprocamente: "Llamamos subjetivización a la implicación de dispositivos tecnológicos, semióticos y sociales en el funcionamiento físico y somático individual. De igual modo, la objetivación se definirá como la implicación mutua de actos subjetivos en el curso de un proceso de construcción de un mundo común. Subjetivación y objetivación son entonces dos movimientos complementarios de la virtualización. En efecto, al considerar aquello que hacen, ni el sujeto ni el objeto son sustancias, sino nodos fluctuantes de acontecimientos que se intercalan y se envuelven recíprocamente." 
espontánea e ingenua ante el mundo circundante, sin discusión o duda alguna: "Un objeto para Husserl no es lo dado, sino que lo dado está constituido por una génesis de los sentidos" (p. 84). Allí la relación entre el sujeto y el objeto no es inmediata; es mediada por la conciencia. La experiencia aparece como una construcción en la que las formas innatas de percepción, de comprensión y los marcos conceptuales aprendidos configuran el lebenswelt o mundo de la vida (Churchland, 1999). En esta línea, y tal como sugiere Ihde (2005), se deben analizar las interrelaciones dinámicas entre seres humanos incorporados en el marco de un mundo concreto; mundo entendido como rango de posibilidades que actúa sobre los seres humanos.

Frente al realismo ingenuo que ve a los objetos digitales como simples símbolos que aparecen en el mundo, o simples herramientas que están allí para su uso, es preciso comprender que las relaciones internas de estos objetos están materializadas y codificadas y que afectan la visión de mundo. Si, como afirman Lovink y Hui (2016:5), "the digital is the capacity to process data", entonces surge con los datos un elemento a reconsiderar en esta posible nueva ontología de carácter relacional. Una advertencia que recupera un posicionamiento no tecnofóbico exige no ver en los datos al enemigo. Si estos han sido objeto de estudio en la historia de las humanidades, hoy requieren profundizarse? . Aunque se apele a su raíz latina como "cosa dada", los datos que interesan no son los datos sensoriales, ni tampoco la perspectiva heideggeriana del darse como modo de estar-juntos. Es ese significado adicional que produjo una transformación material, surgida en el siglo XX, que lo concibe como información computacional capaz de ser transmitida y almacenada:

La importancia de la nueva técnica de procesamiento de datos que ahora llamamos lo digital no consiste solo en que podemos procesar grandes cantidades de datos con computadoras, sino también en que al operar con datos el sistema puede establecer conexiones y formar una red de datos que se extiende de plataforma a plataforma, de base de datos a base de datos... (Hui, 2017: 89).

Esta operación con los datos y algoritmos configuran una experiencia del mundo ${ }^{10}$ que Stiegler, en una relectura de Husserl y centrando su atención en los

\footnotetext{
9 Hui lo afirma como la tarea de las humanidades digitales frente al Big Data. Véase: https://www.eflux.com/journal/78/82706/digital-objects-and-metadata-schemes/.

${ }^{10}$ Sadin (2018) expresa esta situación en el marco de una economía del dato y de la silicolonización del mundo, como un modelo civilizatorio de "acompañamiento algorítmico de la vida destinado a ofrecer a cada ser o entidad, y en todo momento, el mejor de los mundos posibles” (2018: 26).
} 
soportes de memoria, considera el flujo de la conciencia en un marco de temporalidad que se percibe dependiendo constitutivamente por soportes técnicos, por procesos de retención y protención. La protención terciaria aparece como esa capacidad de predicción y anticipación de los algoritmos complementando el concepto de retención terciaria de Stiegler:

Las retenciones son lo retenido o recogido por la consciencia. El término proviene de Husserl, pero las retenciones terciarias son propias de la filosofía de Bernard Stiegler. Las retenciones son selecciones: en el flujo de la conciencia que uno es, no nos es posible retener todo, lo que retenemos es lo que somos, pero lo que retenemos depende de aquello que ya hemos retenido. La especie humana, originalmente protésica, dispone de una tercera memoria, que no es genética ni epigenética: el medio epifilogenético, como conjunto de retenciones terciarias que configuran dispositivos retencionales (2014: 250).

Estas retenciones terciarias propias del ámbito de la técnica serán los soportes materiales, los lugares en los que se deposita la memoria individual que de un modo extra-orgánico se asientan en la materia inorgánica organizada. Estos soportes poseen propiedades materiales que habilitan la conservación de contenidos en el tiempo, modifican la experiencia temporal y espacial de almacenamiento de información, el proceso de acceso a ella y cómo se produce, ampliando capacidades específicas del ser humano. Los soportes, que ahora sufren transformaciones físicas y son dinámicos, permiten no solo el movimiento de los mismos soportes sino también de sus contenidos. Las operaciones de inscripción y transmisión de datos son operaciones que reemplazan la mediación humana por acciones de una técnica que opera cada vez en menor tiempo, evitando retrasos en el procesamiento de acontecimiento-registro-recepción (Vaccari, 2010). De esta manera, lo que Lovink y Hui (2016) denominan protención terciaria, se amplifica en los grandes datos con su capacidad de predicción y anticipación ${ }^{11}$ y con ello, una nueva forma de conceptualizar el tiempo y relacionarnos con él.

${ }^{11}$ Stiegler, B, y Petit, V: Pharmacologie du Front national: Suivi du Vocabulaire d'Ars Industrialis. Flammarion, 2013. 


\section{La digitalización del mundo y el régimen dual de la experiencia humana}

La pregunta simondoniana sobre aquello que provocó la oposición entre cultura y técnica reconociendo en el objeto técnico un extranjero ${ }^{12}$, requiere en el caso de los objetos digitales, un tratamiento particular. La historia muestra que esta oposición se ha mantenido con más énfasis a partir del desarrollo de las tecnologías digitales. Lo digital como cuerpo disociado se sustancializa tanto por aquellas tendencias tecnófilas como por aquellas incapaces de establecer un marco de interpretación conceptual. Es preciso comprender que este "esencialismo fascinado" generó un distanciamiento de las condiciones de formación de lo digital (Sadin, 2018: 41-42).

En el contexto de la disolución del sujeto moderno, una nueva antropología a partir de lo digital extiende las facultades del entendimiento y la aprehensión del mundo. Se trata de un acoplamiento entre organismos fisiológicos y códigos digitales conformando una red inestable de capacidades y objetivos otorgados al ser humano y a las máquinas. Sin embargo, la relevancia de esta revolución digital no reside específicamente en la capacidad ampliada de comunicación, en el acceso a diversos servicios online o en la descarga simple de aplicaciones, por ejemplo, al smartphone (hiperconexión, hipermóvil). Sin dudas son prácticas que efectivamente modifican aspectos tecnológicos, económicos, sociales y culturales que prefiguran esquemas de pensamiento y moldean el comportamiento de los individuos. Pero la parcialidad del análisis puede ocultar dos aspectos esenciales en esta revolución: la delegación de la facultad de interpretación y de iniciativa otorgada a la técnica, por un lado, y por otro, la "subjetividad ampliada" (hibridación) acompañada de "protocolos deductivos" que orientan y deciden comportamientos individuales y colectivos (Sadin, 2017).

Escapando a los determinismos tecnológicos, es necesario pensar la idea de una relación puntual y más flexible entre tecnologías digitales y nuestra experiencia del mundo. Ellas aparecen más como plataformas con infinitas posibilidades de combinaciones, rupturas y desarrollos que como una fuerza determinante. No obstante, el objeto digital se torna invisible, mostrando solo los efectos de la pantalla para un usuario, cuya relación con los dispositivos

\footnotetext{
12 Dirá Simondon: "La máquina es el extranjero; es el extranjero en el cual está encerrado lo humano, desconocido, materializado, vuelto servil, pero mientras sigue siendo, sin embargo, lo humano" (Simondon, 2007: 31).
} 
técnicos prioriza una mera lógica consumista y/o de facilidad de acceso a la información que no busca problematizarse demasiado ${ }^{13}$. En este caso, se concibe al objeto digital simplemente como un nuevo medio en el cual las personas se desenvuelven cotidianamente. La situación cambia, y el conflicto aparece, cuando se advierte la tendencia a delegar en la máquina ciertos procesos cognitivos complejos que ponen en riesgo la capacidad de juzgar y actuar libremente, tal como lo expresa Sadin $(2017,2018)$ o, directamente perdiendo esas mismas capacidades $^{14}$ (Berry, 2011a). De allí deviene una pregunta necesaria para comenzar a indagar y explorar en el papel de los objetos digitales: ¿'Todo proceso de digitalización causa un empobrecimiento de la experiencia humana? ${ }^{15}$

Si se piensa a partir del carácter "aumentado" de la realidad la respuesta sería negativa. La tendencia de la tecnología contemporánea apunta a un presente vivido intensamente ofreciéndole, supuestamente, un presente "enriquecido". En este marco, la instauración de una nueva subjetividad bibrida o bumanidad aumentada fija la experiencia orientada ahora por ecuaciones algorítmicas nacidas de procesos lógicos y abstractos constituyendo el revés de su moneda, la cara de una gubernamentalidad algorítmica del mundo, incapaz de dar cuenta de la multidimensionalidad constitutiva de la vida y lo real (Sadin, 2018: 302-303). El dominio algorítmico se expresa en su penetración al campo de la decisión autónoma y la profundización o la ampliación de las capacidades sensitivas, perceptivas, y cognitivas del hombre. Es decir, un régimen dual de la experiencia permitido por "la capacidad de los robots" y el saber algorítmico generalizado. "Son juegos de entrelazamientos que oscilan de facto entre la adquisición de una

\footnotetext{
${ }^{13}$ Aquí es posible advertir, siguiendo a Carr (s/f), como la Red promueve un estilo de lectura en el que prevalece la inmediatez y la eficiencia. Así, los sujetos se transforman en meros decodificadores de información, y sus capacidades de concentración y contemplación se hallan socavadas. En consecuencia, la Red se encarga de conformar el proceso de pensamiento de los sujetos.

${ }^{14}$ Berry lo ejemplifica citando en el caso de los cambios en los procesos de investigación: "Mientras algunos denuncian la pérdida de las técnicas y habilidades pertenecientes a viejas tradiciones de búsqueda, centradas en una "lectura cercana", otros han abrazado las llamadas humanidades digitales, que han sido fuertemente asociadas con el uso de métodos de asistencia computarizadas al académico" (Berry, 2001a:1).

${ }^{15}$ Esta pregunta por la experiencia se puede asociar a otra que formulara Lévy (2007): ¿Un proceso de digitalización implica una desmaterialización de la información? En algún sentido la respuesta es afirmativa, fundamentalmente comprobado en aquellos objetos que no nacieron digitales y que luego se digitalizan (como por ejemplo una fotografía bidimensional). Pero también en el caso de objetos ya nacidos digitales como un programa (software). Esta digitalización no puede existir sin soporte físico, ocupa un lugar en el espacio, precisa de energía física para grabarse, se "almacena" y se recupera. Estos objetos digitales pueden expresarse visiblemente en un número indefinido de posibilidades según la situación o los requerimientos de los usuarios y programadores. Por eso no son solo herramientas "para producir algo", operan virtualizando la información. Quizás sea más adecuado hablar de desterritorialización o desustanciación.
} 
subjetividad altamente informada y la modificación de las decisiones por obra de algoritmos con poderes eminentemente seductores" (Sadin, 2017: 84-85).

En este contexto que puede ser caracterizado como de obsolescencia del hombre y de lo que podría llamarse, de un soft totalitarismo digital, se produce esa separación del derecho de actuar con conciencia y libertad plena. Aquí "la naturaleza de lo digital se modifica. Hasta este momento estuvo estructurada en función de garantizar principalmente la gestión de datos; ahora está dotada de una actitud interpretativa y decisional" (Sadin, 2018: 36-37).

En otra línea, además de descubrir esta implicancia en la conformación de una subjetividad bibrida, se incluye la desconfianza hacia la mediación de dispositivos digitales en la visión de la realidad y la forma de un pensar computacional que nos convertiría en objetos (no sujetos) de esta tecnología con la aparente pérdida de libertad psicológica:

This would represent the final act in a historical process of reclassification of entities from persons to objects - potentially, dasein becoming an entity amongst entities, an stream amongst streams - with challenging political and cultural implications for our ability to trace the boundary between the human and non-human. This, of course, returns us to the questions raised at the beginning of the book regarding humanity's ontological precariousness. In allowing the computational to absorb our cognitive abilities, off-loading the required critical faculties that we presently consider crucial for the definition of a life examined, we pay a heavy price, both in terms of the inability of computational methods to offer any way of engaging with questions of being, but also in the unreadiness-to-hand that computational devices offer as a fragmentary mediation of the world (Berry, 2011b: 168).

Este pensar de forma computacional ${ }^{16}$ es lo que estaría en cuestión al vislumbrar una mediación fragmentada del mundo, ubicando al sujeto, ahora,

\footnotetext{
${ }^{16}$ Los orígenes de las críticas al pensar computacional, calculante y con pretensiones de cuantificar el universo son posibles de hallarlos en la propuesta de Heidegger (1994), quien, desde su ontología y consecuente pregunta por el ser, da cuenta de las diferencias entre el pensamiento calculado y el reflexivo (imprescindible para dar cuenta de lo expresado por Berry y Sadin en el marco de las tecnologías digitales). El hombre actual, esgrime el filósofo alemán, se halla en fuga de pensar. Al respecto resalta: "Solo aquello que, sabiéndolo o no, poseemos, podemos perderlo, o como se dice quedarnos sin ello" (Heidegger, 1994: 23). En el marco de la digitalización de la vida cotidiana, la carencia real de libertad psicológica advertida por Berry da cuenta de esa pérdida real de pensamiento. Y siguiendo a Marcuse (1993), las personas, por ignorancia o derrotismo, reniegan de esta falta de pensamiento reflexivo o directamente, no son capaces de advertirlo. En su obra El Hombre Unidimensional, Marcuse discute en muchos pasajes el papel acomodaticio de la razón frente al orden imperante en el marco de
} 
como objeto de la tecnología digital. Se observa el agravamiento de la pérdida de capacidades humanas a raíz de los procesos de innovación y producción de dispositivos digitales. Mario Sei, en su artículo Técnica, Memoria e Individuación: la perspectiva de Bernard Stiegler, manifiesta:

Acceder a los flujos de los acontecimientos informativos que, en los espacios virtuales de los datos, se producen a millones por segundo, significa para las conciencias poseer una serie de interfaces (una pantalla de televisión, un ordenador o un teléfono portátil) las cuales son a su vez productos industriales, sujetas por tanto al ritmo de la innovación permanente, lo que no hace sino agravar la pérdida general de inteligibilidad de todo lo que sucede, sin que sepamos hasta qué punto es fabricado, falsificado o registrado en las distintas pantallas de refracción que cuentan en directo lo real (2004: 5).

La codificación de la realidad vuelve opaca esta inteligibilidad, oculta y hace imperceptible su real potencia que modula el curso de la vida. La consideración de una dimensión imperceptible y potencia interpretativa de los procesadores configuran esta soberanía de la técnica "no como una fuerza que coacciona, prescriptiva o esclavizante, sino como una entidad impersonal, multiforme... aportando en parte a hacer retroceder el ejercicio de nuestra facultad de juicio en el aquí y ahora" (Sadin, 2017: 86). Se evidencia así, una constante delegación y dependencia a los poderes de la inteligencia computacional, en donde la algoritimización configura nuestras acciones y modos de desenvolvernos en la vida cotidiana. Con el propósito de ilustrar y advertir el papel actual de la $\operatorname{Red}^{17}$ se recuerda la profecía de Stanley Kubrick: "según confiemos en las computadoras para mediar nuestra comprensión del mundo es nuestra propia inteligencia la que se aplana hasta convertirse en inteligencia artificial" (Carr, s/f: 9).

la constante proliferación de necesidades falsas (cuyo contenido y función social son determinados por poderes externos) conforme al desarrollo de las sociedades industriales avanzadas.

${ }_{17}$ Carr enfatiza en las ambiciones y propósitos que guían el quehacer de Google para dar cuenta de las implicancias de la Red en el comportamiento de los sujetos inmersos en esta sociedad digitalizada. Al respecto enuncia: "La compañía ha declarado que su misión es "organizar la información mundial y hacerla universalmente accesible y útil". Procura desarrollar "el motor de búsqueda perfecto" al que define como algo que "entiende exactamente lo que uno quiere decir y le devuelve exactamente lo que desea. Al entender de Google, la información es un tipo de producto, un recurso utilitario que puede extraerse y procesarse con eficiencia industrial" (s/f: 6). No menos importante, aduce que Internet es un sistema de computación inconmensurablemente poderoso que está subsumiendo la mayoría de nuestras otras tecnologías intelectuales. 
De todas maneras, los caminos frente al interrogante planteado en los párrafos precedentes pueden ser dos: el consumo de información personalizada en cada esfera de la vida, disminuyendo nuestras capacidades cognitivas y volitivas asistidos por "la magia de los algoritmos" y ampliando cierta "pereza natural". Esto se complementa con lo expresado por Sadin en lo que denomina la aparición del sindrome del tiempo real o Sindrome Sherlock, como un "desfasaje que se siente entre la sensación de dominar secuencias cada vez más extendidas de lo cotidiano, y la de constatar lo vano de ese poder en comparación con la dificultad para realizarse plenamente a partir de las propias facultades" (2018: 206). Y el otro camino: beneficiarse con la recepción de nuevos saberes y en paralelo, enriquecer nuestra experiencia y capacidades tomando conciencia de nuestra nueva condición humana (Sadin, 2017).

La alienación, no en el sentido marxista del término, sino más específicamente como instancia de desvinculación de una cultura técnica, se produce cuando no se sabe o no se puede interpretar este interrogante sobre la experiencia y su vínculo mediatizado con lo real, y además cuando se considera al hombre como un simple usuario que delega su capacidad en estos objetos digitales:

Las ideas de servidumbre y liberación están demasiado ligadas al antiguo estatuto del hombre como objeto técnico como para poder corresponder al verdadero problema de la relación del hombre y de la máquina. Es necesario que el objeto técnico sea conocido en sí mismo para que la relación del hombre con la máquina se convierta en válida y estable: de allí la necesidad de una cultura técnica (Simondon, 2007: 102).

Simondon advierte que una técnica excluida de la cultura produce una separación irreconciliable entre lo natural y lo artificial. Paradójicamente una técnica pensada y producida por el hombre mismo, resulta ajena a él. Una relación armónica entre cultura y técnica sólo será posible si se realiza una adecuada comprensión de los objetos técnicos, y en nuestra actual preocupación, también de los objetos digitales.

\section{Consideraciones finales}

Abandonando toda posición realista ingenua, sustancialista, o instrumental sobre la técnica, nos aproximamos a una reflexión sobre los objetos digitales que conlleva una perspectiva relacional, ubicándolos y configurando una experiencia 
del mundo, con experiencias generalizadas de juegos combinatorios ilimitados y exponenciales, tanto en el almacenamiento como en el tratamiento y los tipos de datos que se utilizan. Retención y protención terciaria permiten comprender la amplificación de la gran cantidad de datos informacionales con su capacidad de predicción y anticipación estableciendo una nueva forma de pensar y relacionarnos con el tiempo. De esta forma, Hui observa que una ontología digital vinculada únicamente al código binario, a bits y bytes, no es suficiente para comprender nuestra experiencia vital en medio de la aceleración tecnológica. Para lograrlo, debiéramos abandonar lo digital en términos de abstracciones (información, datos, metadatos), y abrazar la posibilidad de pensar la materialidad de estos objetos desde un abordaje que priorice el aspecto relacional.

En esta línea, y siguiendo la perspectiva del actor-red, establecer de manera apriorística la prevalencia de las intenciones- en términos de agencia- de los sujetos frente a los objetos, trae ciertas dificultades para reconocer esta agencia en los objetos digitales, al ser tratados como objetos pasivos y subsidiarios en la actual digitalización de la vida humana.

Por otra parte, pensar en el modo de existencia de los objetos digitales también lo es pensar en una nueva cultura de la técnica. Tanto un objeto técnico, como un objeto digital, concretizan esquemas operativos y de pensamiento con una finalidad específica llevando en sí algo del ser que lo produjo. Un usuario tendría que ser capaz de establecer una relación de diálogo con el objeto poseedor de una información esencial que debería ser comprendida. Se trata del conocimiento en sí mismo del objeto. Cuando esta relación usuario-objeto no sucede, deriva en una alienación fundamental; se cae en posiciones instrumentalistas, y en consecuencia, el objeto aparece como un medio o como una herramienta que puede ser útil o peligrosa (Tula Molina y Giuliano, 2015).

Se necesita una cultura técnica que se aproxime, al menos, a reconocer estos nuevos objetos digitales en sí mismos y su implicancia en el devenir de nuestro presente. En una época signada por una alienación devenida por la opacidad estructural que presenta la tecnología en el marco de la proliferación de estos objetos, se precisa generar condiciones que promuevan la formación de ciudadanos tecnológicos, y no de meros consumidores. Sintéticamente, esto responde a que la relación tecnológica actual que los consumidores establecen con ella, se halla circunscripta a los esquemas técnicos promovidos por el aparato 
industrial. En este marco, una bildung digital (Berry, 2011: 26) es necesaria, no como propuesta formativa en competencias instrumentales, o en abordajes teóricos acumulativos, sino como una instancia reflexiva que permita, por un lado, a los sujetos unificar la producción acelerada y creciente de información (digital) y por otro, a comprender los nuevos métodos y prácticas de lectura crítica con códigos, visualización de datos y narrativas. Esta misma instancia de una bildung digital, lejos de los objetivos que persiguen empobrecer y encuadrar la experiencia humana, debería ser un movimiento de defensa de lo sensible, como condición de la experiencia y el uso de la propia libertad de juicio y acción revalorizando la multidimensionalidad del ser humano (Sadin, 2018).

En este escenario, la lectura de Berry muestra la pérdida de capacidades humanas delegadas en los objetos digitales y a la naturalización de una racionalidad específica (la computacional) convertida en única, determinando la construcción de una idea de mundo que descarta otras visiones que no se encuadren en una dimensión cuantitativa. De sostenerse esta mirada, la separación entre pensar y calcular es un hecho consumado.

La humanidad aumentada, la humanidad asistida algorítmicamente, es en alguna medida la que muestra los intentos por pensar y problematizar esa escisión. Es la invitación a reflexionar en esta nueva condición humana más allá del rechazo, pensándola en este "horizonte híbrido en devenir", que contemple la presencia de los objetos digitales como parte esencial en la construcción de la realidad.

Sin embargo, Sadin parece tomar en este último tiempo una posición más radical frente a los efectos de esta sociedad aumentada. Con el sindrome del tiempo real, se constata el empobrecimiento de nuestra experiencia humana. Afuera quedan las experimentaciones vitales, los caminos inciertos, las decepciones, los éxitos y fracasos que, ahora mediatizados por algoritmos, nos constituyen de otro modo, debilitando nuestras capacidades para entendernos con lo real y con el ejercicio de nuestras facultades sensibles.

Una nueva ontología que se desvíe de posiciones instrumentalistas y sustantivistas y que tome a estos objetos, o que problematice, por ejemplo, el software cultural, se torna más que necesario. Un humanismo que, en el tipo de relación que construye con la técnica, se limite al uso instrumental o de sometimiento maquínico, no alcanza a interpretar las características de esta época provocando un desconocimiento e incomprensión de su funcionamiento y de 
cuánto de lo humano se juega allí mismo (Blanco, 2014). Pero tampoco sirve ese humanismo optimista y acrítico que se ampara en una técnica predictiva y adaptativa que nos "liberaría", del azar, de lo imprevisto. Un juego, con respecto a la técnica, de necesario repliegue y distanciamiento, pero también de una inmersión, de una implicación responsable y deseada.

Desde el posthumanismo cínico ${ }^{18}$ se recuerda que "el destierro de los hábitos de apariencia humanística es el acontecimiento lógico principal de nuestro tiempo..." (Sloterdijk, 2001: 20), y para pensar a los objetos digitales, es necesario recordar esta afirmación. También preguntar si el hombre, en este proceso de informatización de la sociedad, descubre "si lo que puede y hace tiene que ver con él mismo" porque la actual lógica y ontología humanista es incapaz de interpretar adecuadamente estas nuevas entidades digitales en donde categorías como "cuerpo y alma, espíritu y materia, sujeto y objeto, libertad y mecanismo, no puede ya habérselas con entidades de este tipo" (2001: 22).

\section{Referencias}

Berti, A. y Blanco, J. (2013). ¿Objetos digitales? IV Coloquio Internacional de Filosofía de la Tecnologia: Tensiones, continuidades y rupturas. Universidad Abierta Interamericana. Recuperado de https://www.aacademica.org/agustin.berti/42.pdf.

Berti, A. (2017). La integridad de los replicantes. Medio asociado y límites en los objetos digitales. En: Lawler, D. et al (comps.). La técnica en cuestión. Artificialidad, cultura material y ontología de lo creado. Buenos Aires: Teseo: Universidad Abierta Interamericana.

\footnotetext{
${ }^{18}$ Para futuros trabajos sobre las relaciones entre cultura y técnica puede resultar interesante discutir algunas aseveraciones de Luis García, su crítica a Sloterdijk y sus afirmaciones sobre una política de la indeterminación en un posthumanismo radical: "En este sentido, postulamos que, en la actualidad, la disputa real no se da entre humanismo y posthumanismo... sino entre estas dos fundamentales "alternativas de lo posthumano": el posthumanismo cínico de la apología de la subjetividad del rendimiento y de la sociedad de control, por un lado, y el posthumanismo de la experimentación con las formas de la artefactualidad radical de la subjetividad y de la comunidad" (2016: 29).
} 
Berry, D. (2011a). Humanidades Digitales: Primera, Segunda y Tercera Ola. Recuperado de: http://catedradatos.com.ar/media/4.-David-Berry-HumanidadesDigitiales.pdf

(2011b). The computational turn: Thinking about the digital humanities. Culture Machine, 12. Recuperado de: http://www.culturemachine.net/index.php/cm/article/view/440/470

(2011). The Philosophy of Software. Code and Mediation in the Digital Age. New York: Palgrave Macmillan.

Blanco, J. (2014). Pensar y calcular. Revista Nombres. (28), 213-229.

Carr, N. (s/f). ¿Qué le está haciendo Internet a nuestros cerebros? Recuperado de

http://asociacioneuc.org/documentos/docsEUCs/62EUCNicholasCarr.p df.

Clark, A. y Chalmers, D. (2011). La mente extendida. Oviedo: KRK Ediciones.

Churchland, P. (1999). Materia y Conciencia. Introducción contemporánea a la Filosofía de la mente. Barcelona: Gedisa.

Galimberti, U. (2001). Psiché y Techné. Artefacto. Pensamientos sobre la técnica. (4). [Traducción: Flavia Costa. De Galimberti, Umberto. Psiché e Techné. L'uomo Milano, Feltrinelli, 1999]. Recuperado de http:/ / postitulo.sociales.infd.edu.ar/archivos/repositorio/250/398/TSMC _Clase-1_Galimberti.pdf.

Feenberg, A. (2005). Teoría crítica de la tecnología. Revista CTS, 2, (5), 109-123.

Fredkin, E. (2003). An Introduction to Digital Philosophy. Carnegie Mellon University.

(2012). Transformar la realidad. Una nueva visita a la teoría crítica. Buenos Aires: Universidad Nacional de Quilmes.

García, L. (2016). Técnica, posthumanismo y experiencia. Revista Nombres. Año $\mathrm{XXV},(30), 21-40$.

Heidegger, M. (1994). Serenidad. Bogotá: Revista Colombiana de Psicología. 
Aproximaciones a la problematización del objeto digital en el marco de la digitalización de la vida

Hui, Y. (2016). On the Existence of Digital Objects. Minneapolis: University of Minnesota Press.

Husserl, E (2008). Crisis de las ciencias europeas y la fenomenología trascendental. Buenos Aires: Prometeo Libros.

(2017). ¿Qué es un objeto digital? Virtualis, 8, (15), 81-96.

Ihde, D. (2005). La incorporación de lo material. Fenomenologia y filosofia de la tecnología. Revista CTS, (5), 153-166.

Latour, B. (2008). Reensamblar lo social. Una introducción a la teoría del actor-red. Buenos Aires: Manantial.

Lévy, P. (1999) ¿Qué es lo virtual? Buenos Aires: Paidós.

Lovink, G. y Hui, Y. (2016). Digital Objects and Metadata Schemes. Revista e-flux, (78). Recuperado de https://www.e-flux.com/journal/78/82706/digitalobjects-and-metadata-schemes/

Manovich, L. (2008). El software toma el mando. Traducido por Adrián Yalj y Sofía Alamo (Cátedra Procesamiento de Datos, Carrera de Ciencias de la Comunicación, Facultad de Ciencias Sociales, Universidad de Buenos Aires). Recuperado de https://catedradatos.com.ar/media/Lev-ManovichEl-Software-toma-el-Mando-2008-Introducci\%C3\%B3n-_Estudios-deSoftware-para-principiantes-.pdf

Marcuse, H. (1993). El hombre unidimensional. Ensayo sobre la ideología de la sociedad industrial avanzada. Planeta Agostini.

Montfort, N. (2004). Continuous Paper: The Early Materiality and Workings of Electronic Literature. Recuperado de http://nickm.com/writing/essays/continuous_paper_mla.html

Rodríguez, P. (2012). Historia de la Información. Bs. As.: Capital Intelectual.

Sadin, E (2017). La humanidad aumentada: la administración digital del mundo. Buenos Aires: Caja Negra. 
(2018). La silicolonización del mundo. La irresistible expansión del liberalismo digital. Buenos Aires: Caja Negra.

Sei, M. (2004). Técnica, Memoria e Individuación: la perspectiva de Bernard Stiegler. LOGOS. Anales del Seminario de Metafísica, (37), 337-363.

Simondon, G. (2007). El modo de existencia de los objetos técnicos. Buenos. Aires: Prometeo.

(2015). La individuación a la luz de las nociones de forma y de información. Bs. As.: Cactus.

Sloterdijk, P. (2001). El hombre operable. Notas sobre el estado ético de la tecnología génica. Revista Artefacto. Pensamientos sobre la técnica, (4), 20-29.

Stiegler, B. (2002). La técnica y el tiempo 1. Elpecado de Epimeteo. España: Hiru.

Tula Molina, F. y Giuliano, H. (2015). Hacia una nueva cultura de la técnica: horizonte ingenieril y horizonte filosófico. En Blanco, J., Parente, D. y Rodríguez, P. (comps.) Amar a las máquinas. Cultura y técnica en Gilbert Simondon. Buenos Aires: Prometeo.

Vaccari, A. (2010). Vida, técnica y naturaleza en el pensamiento de Gilbert Simondon. Revista CTS, (5), 173-185. 